\title{
Increasing evidence of combinatory variant effects calls for revised classification of low-penetrance alleles
}

\author{
Nicholas K. Wang, MHS ${ }^{1}$ and John P. W. Chiang, PhD, FACMG ${ }^{1}$ \\ Genetics in Medicine (2019) 21:1280-1282; https://doi.org/10.1038/s41436-018-0347-3
}

In the era of precision medicine, variant interpretation is arguably the most challenging aspect of molecular diagnosis. Despite continuous and rapid advances in the field, many genes still do not have well-established genotype-phenotype correlations. On a variant level, pathogenicity characterizations have mostly relied on qualitative descriptors within a Mendelian framework while less discrete effects have been difficult to quantitate. ${ }^{1}$ Attempts to aggregate variant information across large cohorts have given us useful metrics such as population allele frequencies; however, these can be difficult to make inferences from due to variation across ancestral groups ${ }^{2}$ or allele association with conditions of varying clinical presentation. $^{3-8}$ Variants with hypomorphic effects or incomplete penetrance further complicate classification with less transparent conditional effects leading to frequent conflicting interpretations. ${ }^{9}$

Here, we present three well-documented examples of low-penetrance alleles, beginning with a TYR variant described in a case study by our group in $2008^{3}$. Since then, this quantitative effect concept has been continually observed in our clinical diagnostics work as well as corroborated by various groups across a spectrum of conditions. $^{5-8}$ These examples serve to illustrate the difficulty of variant interpretation for low-penetrance alleles and the pressing need for their precise, clinically informative classification in combination with current American College of Medical Genetics and Genomics/ Association for Molecular Pathology (ACMG/AMP) guidelines.

Variant information and reported interpretations taken from dbSNP (https://www.ncbi.nlm.nih.gov/ projects/SNP/) and ClinVar (https://www.ncbi.nlm.nih. gov/clinvar/) at the time of writing. The authors would like to thank the Genome Aggregation Database (gnomAD, http://gnomad.broadinstitute.org/) and the groups that provided exome and genome variant data to this resource for the referenced population allele frequencies. ${ }^{2}$
(1) TYR (OCA1); NM_000372.4:c.1205G>A (p.Arg402Gln); rs1126809

ClinVar Variation ID: 3779

Submitted Interpretations: Benign (2), Likely Benign (1), Likely Pathogenic (1), Pathogenic (3), Uncertain Significance (1), Other (1), Association (2), Risk Factor (1)

Oculocutaneous albinism type I (OCA1) is an autosomal recessive condition attributed to pathogenic variants in $T Y R$. Milder presentations of the condition have been observed with only one classic pathogenic TYR variant in trans with the Arg402Gln allele. Similarly, a milder form of OCA1 without noticeable cutaneous effects, autosomal recessive ocular albinism (AROA), has been linked to compound heterozygosity of TYR pathogenic variants with Arg402Gln. ${ }^{3}$ Although this allele has been shown to encode for a functionally impaired tyrosinase, its high population allele frequency (gnomAD AF 0.17640 ) and presence of unaffected homozygotes ${ }^{4}$ have made classification of clinical significance difficult. Segregation studies of the Arg402Gln allele in OCA1 families have also revealed cases of unaffected individuals harboring this compound heterozygous combination suggesting that while Arg402Gln can act as a hypomorphic allele, its presence in trans with a TYR mutant allele is not necessarily sufficient for phenotypic presentation. ${ }^{4}$ Further investigation, such as through case-control association studies, will be needed to uncover additional modifying factors that may contribute to the AROA condition.

(2) GALT; NM_000155.3:c. -119_-116delGTCA; rs11103 3640

ClinVar Variation ID: 25111

Submitted Interpretations: Benign (2), Pathogenic (3)

Classical galactosemia is an autosomal recessive condition characterized by deficient GALT enzymatic activity due to pathogenic variants in GALT. A milder form of

${ }^{1}$ Molecular Vision Laboratory, Hillsboro, OR, USA. Correspondence: John P. W. Chiang (jchiang@mvisionlab.com) 
the condition was originally associated with the Duarte allele (p.Asn314Asp) where the impact on GALT activity is less pronounced than causal classic galactosemia alleles. Later studies revealed the causal variant for this effect to be a 4-bp deletion in the $5^{\prime}$ region of GALT in linkage disequilibrium with Asn314Asp with the combined haplotype termed D2 $2^{5}$ Functional studies confirmed this effect demonstrating that this 4-bp deletion interferes with transcription factor binding and reduces GALT promoter activity. ${ }^{6}$ While classical galactosemia follows expected recessive inheritance patterns, the hypomorphic effect of the D2 allele leads to D2 homozygotes with $\sim 50 \%$ reduced GALT activity but healthy clinical presentation. ${ }^{6}$ Duarte galactosemia patients are compound heterozygous for the D2 allele and a classic pathogenic GALT allele with $\sim 25 \%$ normal GALT activity and mild to asymptomatic presentation. ${ }^{5,6}$ This variable clinical presentation and incidence of up to 1 in 4000 live births ${ }^{5}$ along with the presence of unaffected homozygotes distinguishes the pathogenicity of this variant from other pathogenic variants-a difference easy to overlook if only a "pathogenic" label is annotated or referenced.

(3) RBM8A; NM_005105.4:c.-21G >A; rs139428292

ClinVar Variation ID: 30464

Submitted interpretations: Likely Pathogenic (1), Pathogenic (6), Other (1)

RBM8A; NM_005105.4:c.67+32G>C; rs201779890

ClinVar Variation ID: 30465

Submitted interpretations: Likely Pathogenic (3), Pathogenic (3)

Thrombocytopenia-absent radius (TAR) syndrome is an inherited genetic condition characterized by reduced platelet count and absence of the radius in the forearm. ${ }^{7}$ The condition was originally associated with a 200-kb deletion on chromosome 1q21.1 found across an entire 30-patient cohort and absent in controls. The authors hypothesized that TAR syndrome is associated with this deletion, however, only in combination with an additional unknown factor due to its recessive inheritance pattern and presence of unaffected carriers. Sequencing of the coding regions of ten genes within the deleted region on the opposite allele was inconclusive. ${ }^{8}$ Exome sequencing later revealed two low-frequency singlenucleotide polymorphisms (SNPs) in the 5'UTR (c. $-21 \mathrm{G}>\mathrm{A}$; gnomAD AF 0.01812) and first intron (c.67+32G $>$ C; gnomAD AF 0.00584) of RBM8A identified in 39 and 12 patients of 53, respectively. In vitro studies showed reduced $R M B 8 A$ promoter activity from both SNPs suggesting a hypomorphic mechanism where compound heterozygosity of a null allele with $\mathrm{c}$. $-21 \mathrm{G}>\mathrm{A}$ or $\mathrm{c} .67+32 \mathrm{G}>\mathrm{C}$ is likely causative for TAR syndrome. Furthermore, no significant variants were found in linkage equilibrium with either SNP, supporting their role as the second causative variant.' Of particular interest, these two variants both appear in ClinVar with concordant interpretations of "pathogenic" or "likely pathogenic" despite relatively high population allele frequencies and the presence of homozygotes in the gnomAD cohort (63 c. $-21 \mathrm{G}>\mathrm{A}$ homozygotes; 12c.67+32G $>$ C homozygotes). ${ }^{2}$ Under ACMG/AMP guidelines, this evidence would support the BS1 and BS2 criteria for benign classification ${ }^{1}$ highlighting the room for ambiguity that exists in current interpretations.

While publicly available, aggregated sources of variant interpretation, such as ClinVar, are invaluable in the mission to understand the genetic factors of human health, the framework in which this information is organized is critical to its utility. How variants are classified and reported must be continually evaluated and adapted to keep up with our expanding knowledge of genetic mechanisms and variant effects. These three examples outline some of the challenges of variant interpretation and the difficulties faced when attempting to classify combinatory variant effects within the current ACMG/AMP guidelines. ${ }^{1}$ While clinical significance categories of "association" and "risk" exist as alternatives to the standard "benign" to "pathogenic" scale, the effects of hypomorphic alleles and other reduced penetrance variants do not necessarily fit their guidance criteria. The "other" category exists as an option to provide additional information in the form of a comment; however, with the increasing awareness of such variants, we believe that a reevaluation of these classifications is warranted.

To this end, we propose the introduction of a new category of clinical significance for these lower-penetrance alleles that fall beyond the current "association" or "risk" classifications. We envision this category to encompass variants with hypomorphic effects and evidence of pathogenicity only in combination with severe loss-of-function alleles thus providing a clear classification for variants such as the three described previously. This category would allow for the conditional nature of these variants to be recognized in the context of a quantitative effect model-distinguished from the probabilistic criteria of the current "likely pathogenic" and "pathogenic" categories. Here, the difference between a variant with strong evidence of a hypomorphic effect can be differentiated from a variant with moderate evidence of deleterious effect. This distinction will facilitate clinical diagnostics in highlighting the combinatory requirements of reduced penetrance alleles without relying on written commentary from ClinVar submitters to clarify the mechanism of pathogenicity.

While the perspectives outlined in this commentary are not new to the field, we hope that the examples presented demonstrate the importance of reduced penetrance alleles and consequently the importance of how we classify and report them moving forward. In response to suggestions from professionals within the field, we will be following the lead of the Clinical Pharmacogenetics Implementation Consortium 
(CPIC) and their effort in standardizing pharmacogenetic terminology ${ }^{10}$ to engage the genetics community through a Delphi method panel in support of developing broadly accepted and applicable classifications appropriate for clinicians and researchers alike. Similarly, this discussion will allow us to revisit the criteria for other clinical significance categories and make revisions or further specifications as the community sees fit. We encourage research groups and clinical labs to continue to submit their results to ClinVar to keep our knowledge of such variants complete and current and we look forward to working with ClinVar and the broader genetics community to better accommodate these submissions.

\section{DISCLOSURE}

The authors declare no conflicts of interest.

\section{REFERENCES}

1. Richards S, Aziz N, Bale $S$, et al. Standards and guidelines for the interpretation of sequence variants: a joint consensus recommendation of the American College of Medical Genetics and Genomics and the Association for Molecular Pathology. Genet Med. 2015;17:405-424

2. Lek M, Karczewski KJ, Minikel EV, et al. Analysis of protein-coding genetic variation in 60,706 humans. Nature. 2016;536:285-291.

3. Chiang P-W, Drautz JM, Tsai AC-H, Spector E, Clericuzio CL. A new hypothesis of OCA1B. Am J Med Genet A. 2008;146A:2968-2970.

4. Oetting WS, Pietsch J, Brott MJ, et al. The R402Q tyrosinase variant does not cause autosomal recessive ocular albinism. Am J Med Genet A. 2009;149A:466-469.

5. Carney $A E$, Sanders RD, Garza $K R$, et al. Origins, distribution and expression of the Duarte-2 (D2) allele of galactose-1-phosphate uridylyltransferase. Hum Mol Genet. 2009;18:1624-1632.
6. Elsas L, Lai K, Saunders CJ, Langley SD. Functional analysis of the human galactose-1-phosphate uridyltransferase promoter in Duarte and LA variant galactosemia. Mol Genet Metab. 2001;72: 297-305.

7. Albers CA, Paul DS, Schulze $\mathrm{H}$, et al. Compound inheritance of a lowfrequency regulatory SNP and a rare null mutation in exon-junction complex subunit RBM8A causes TAR syndrome. Nat Genet. 2012:44:435-439.

8. Klopocki E, Schulze H, Strauss G, et al. Complex inheritance pattern resembling autosomal recessive inheritance involving a microdeletion in thrombocytopenia-absent radius syndrome. Am J Hum Genet. 2007:80:232-240

9. Yang S, Lincoln SE, Kobayashi Y, Nykamp K, Nussbaum RL, Topper S. Sources of discordance among germ-line variant classifications in ClinVar. Genet Med. 2017;19:1118-1126.

10. Caudle KE, Dunnenberger HM, Freimuth RR, et al. Standardizing terms for clinical pharmacogenetic test results: consensus terms from the Clinical Pharmacogenetics Implementation Consortium (CPIC). Genet Med. 2017;19:215-223.

Open Access This article is licensed under a Creative Commons Attribution-NonCommercial-NoDerivatives 4.0 International License, which permits any non-commercial use, sharing, distribution and reproduction in any medium or format, as long as you give appropriate credit to the original author(s) and the source, and provide a link to the Creative Commons license. You do not have permission under this license to share adapted material derived from this article or parts of it. The images or other third party material in this article are included in the article's Creative Commons license, unless indicated otherwise in a credit line to the material. If material is not included in the article's Creative Commons license and your intended use is not permitted by statutory regulation or exceeds the permitted use, you will need to obtain permission directly from the copyright holder. To view a copy of this license, visit http://creativecommons.org/licenses/by-nc-nd/4.0/.

(c) The Author(s) 2018 